\title{
A look at a Hispanic and African American population in an urban prenatal diagnostic center: Referral reasons, amniocentesis acceptance, and abnormalities detected
}

\author{
Debra Baker, $M S^{1}$, Senait Teklehaimanot, $M P H^{2}$, Rosetta Hassan, $M D^{3}$, and Carol Guze, $P h D^{4}$
}

\begin{abstract}
Purpose: To describe the Hispanic and African-American population referred to our inner city Los Angeles Prenatal Diagnostic Center. To evaluate trends in referral reasons, amniocentesis acceptance, and to assess the number and types of fetal abnormalities found from 1995 to 2001. Methods: A retrospective study using the data from 3085 daily log entries on patients referred for prenatal counseling. The data included race, age, referral reason, amniocentesis decision and results, and fetal abnormalities. Results: The population was $76 \%$ Hispanic and $22 \%$ African American. Most referrals were for advanced maternal age (42\%) and maternal serum screening (28\%). The overall amniocentesis acceptance rate was 52\%; advanced maternal age amniocentesis acceptance rate was $46 \%$, and maternal serum screen positive amniocentesis acceptance rate was significantly higher at $64 \%$. There was a significant difference between the overall amniocentesis acceptance rate for Hispanics (48\%) and African Americans (63\%). There was also a significant downward trend in amniocentesis acceptance between 1995 (63\%) and 2001 (39\%). Amniocentesis acceptance was significantly greater among patients who were $<35$ years of age (65\%) than those who were older (47\%). An incidence of $7 \%$ abnormalities was detected by ultrasound and amniocentesis. Conclusions: Acceptance of amniocentesis in the Hispanic and African American population in our prenatal diagnostic center is significantly lower than what has previously been reported in the literature for Caucasians and reported in California statewide prenatal diagnostic center data for non-MS-AFP. There was a significant decline in amniocentesis acceptance from 1995 to 2001. Our incidence of fetal abnormalities was higher than reported in California statewide data. Genet Med 2004:6(4):211-218.
\end{abstract}

Key Words: amniocentesis acceptance, prenatal minority population, advanced maternal age, triple marker screen, prenatal abnormalities

In the United States and many other countries, pregnant women are routinely offered prenatal diagnostic procedures such as the noninvasive procedures of ultrasound and maternal serum screening. The more invasive procedures of amniocentesis and chorionic villus sampling (CVS) are offered only in specific cases, because they carry a risk of complications. Several studies have looked specifically at the acceptance rate of amniocentesis among pregnant women with a high risk of a

From the ${ }^{1}$ Department of Biology, California State University Dominguez Hills, Carson, and David Geffen School of Medicine, University of California, Los Angeles, California, ${ }^{2}$ Research Centers in Minority Institutions (RCMI), Charles R. Drew University of Medicine and Science, Los Angeles, California; ${ }^{3}$ Department of Obstetrics and Gynecology, King Drew Medical Center, Los Angeles, California; and ${ }^{4}$ Department of Biology, California State University Dominguez Hills, Carson, and Genetics Unit, Department of Obstetrics and Gynecology, King Drew Medical Center, Los Angeles, California.

Carol Guze, PhD, Genetics Unit, Department of Obstetrics and Gynecology, King Drew Medical Center, 12021 S. Wilmington Ave., Los Angeles, CA 90059.

Received: February 16, 2004.

Accepted: April 21, 2004.

DOI: 10.1097/01.GIM.0000132684.94642.AO fetal abnormality (e.g., advanced maternal age, positive maternal serum screen, and abnormal ultrasound). ${ }^{1-9}$ Eight of the studies were done on a mixed but primarily Caucasian population, and of these, only four provided acceptance data by ethnicity. Two were done in the United States and two in Europe. A lower amniocentesis acceptance rate was found in the minority populations compared to the Caucasians: African Americans and other minorities $60 \%$ versus Caucasians $82 \%$, $(N=129),{ }^{1}$ East Indian $25 \%$ versus Caucasian and others $60 \%$, $(N=382),{ }^{2}$ and non-Caucasian $35 \%$ versus Caucasian $67 \%(N$ $=683) .{ }^{3}$ Although a few of these studies included Hispanic and African-American patients, none focused on a primarily Hispanic population, and only one focused on a primarily African American population. ${ }^{4}$ This last study looked at a population of 157 patients, of which $82 \%$ were African American and 18\% were Caucasian. African American acceptance was 58\% and Caucasian acceptance was $72 \%$.

The purpose of our study was to describe and assess the utilization of prenatal diagnosis by the Hispanic and AfricanAmerican population referred to our inner city Los Angeles 
Prenatal Diagnostic Center at King Drew Medical Center. Specifically, we wanted to assess trends in referral reasons and amniocentesis uptake and to also assess the incidence of abnormalities among our population. This population, primarily of Hispanics and African Americans, offered a unique opportunity to establish the rate of amniocentesis acceptance by ethnicity and referral reason and to determine the type and number of fetal abnormalities. We also wanted to compare the rates of amniocentesis acceptance of our population with California statewide rates.

\section{METHODS}

\section{Setting and data collection}

King Drew Medical Center (KDMC), a county teaching hospital located in south central Los Angeles, serves a socioeconomically disadvantaged population. Patients were referred to the Prenatal Diagnostic Center (PDC) by the hospital prenatal clinic, four outlying satellite clinics, and 25 surrounding community clinics located within a 15-mile radius of the hospital. This retrospective study used data from the daily log of 3085 patients referred for prenatal counseling over the seven-year period 1995 to 2001. The data included race, age, referral reason, amniocentesis decision and results, and fetal abnormalities. This study received approval from both the Charles Drew University and California State University Dominguez Hills Institutional Review Boards.

\section{Counseling}

All patients referred to the PDC were counseled specifically concerning their referral reason(s). Before the counseling session, all patients were shown a video describing the various reasons for referral to the Genetic Unit as well as the procedure of amniocentesis.

Before our patients made their decisions about amniocentesis, they received genetic counseling. Each patient was counseled individually (other family members are included at the patient's request). Counseling sessions varied depending on the patient's case, but they were 30 to 45 minutes with twothirds of the session spent reviewing the pregnancy and family history and one-third spent in a discussion of the patient's options. The advanced maternal age women were counseled regarding their age-related risk for chromosomal abnormalities, and those who screened positive for the maternal serum alpha-fetoprotein (MS-AFP) were counseled regarding their individual risks for chromosomal defects and/or neural tube defects, as determined by the California Expanded AFP Screening Program. Women who were referred for other high risk factors, such as abnormal ultrasounds, were also appropriately counseled.

Counseling was done by a Board Certified PhD Medical Geneticist with 14 years of experience in genetic counseling. For all Spanish-speaking patients, the counselor was assisted by an experienced Hispanic translator who is a member of the Genetic Unit staff. Thus, our analysis explores acceptance rates in a fully informed group of women. The majority of our patients participated in California's Medicaid program, MediCal; therefore, inability to pay for care was not a factor for our patients when making medical decisions.

\section{Data analysis}

Descriptive statistics and contingency table analysis were used for analysis of all data. The Chi-square test for trends and Pearson's chi-square statistics were used to assess the significance. A value of $P<0.05$ was considered to be statistically significant. Statistical analyses were performed using the Statistical Program for the Social Sciences (SPSS, version 11.5, 2002, SPSS Inc; Chicago, IL).

\section{RESULTS}

\section{Patient characteristics}

The population consisted of 2343 Hispanics (76\%), 692 African Americans (22\%), 41 others (1.7\%), and 9 of unknown ethnicity $(0.3 \%)$. The "other" category includes Caucasians, Asians, American Indians, and Middle Easterners (Fig. 1). The majority of our Hispanic patients were born in Mexico and did not speak English.

The patients ranged in age from 14 to 52 years. The mean age of the population was 32 years. There was no significant difference between the mean age of Hispanics ( 32 years) and the mean age of African Americans (31years). Of the total population, $52 \%$ was 35 years of age or older, which would be expected because the primary referral reason of the patients is advanced maternal age.

\section{Referral reasons}

The patients were referred to our PDC for genetic counseling and ultrasound for a variety of reasons and some were referred for more than one reason. As shown in Table 1, the majority of referrals, $42 \%(N=1491)$, were for AMA (advanced maternal age, $\geq 35$ years at the time of delivery). The second largest group referred was of patients who had an MSAFP-positive screen result, $28 \%(N=1007)$. The following

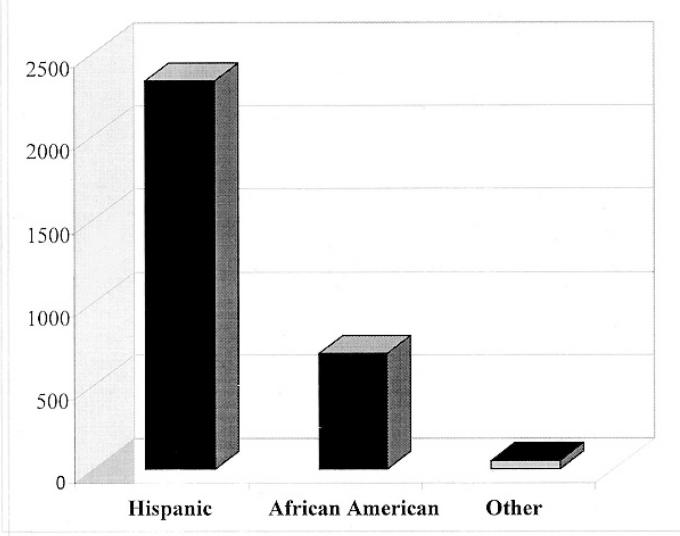

Fig. 1. Prenatal diagnostic center ethnic distribution 1995-2001. 
Table 1

Referral reasons 1995-2001

\begin{tabular}{|c|c|c|c|c|c|c|c|c|}
\hline Referral reason & 1995 & 1996 & 1997 & 1998 & 1999 & 2000 & 2001 & $\begin{array}{c}1995-2001 \\
N(\%)\end{array}$ \\
\hline AMA & 252 & 117 & 226 & 243 & 210 & 263 & 180 & $1491(42)$ \\
\hline MS-AFP & 166 & 79 & 137 & 181 & 125 & 173 & 146 & $1007(28)$ \\
\hline Positive family history & 54 & 27 & 49 & 7 & 50 & 31 & 24 & $292(8)$ \\
\hline Teratogen exposure & 17 & 18 & 32 & 34 & 27 & 43 & 32 & $203(6)$ \\
\hline Diabetes & 16 & 14 & 31 & 16 & 26 & 44 & 26 & $173(5)$ \\
\hline Hemoglobin mutation & 22 & 13 & 24 & 12 & 17 & 30 & 16 & $134(4)$ \\
\hline Other $^{a}$ & 13 & 12 & 22 & 14 & 24 & 14 & 15 & $114(3)$ \\
\hline Pregnancy losses & 15 & 4 & 16 & 12 & 10 & 12 & 10 & $79(2)$ \\
\hline Abnormal ultrasound & 13 & 12 & 13 & 10 & 9 & 7 & 14 & $78(2)$ \\
\hline Total & 568 & 296 & 550 & 579 & 498 & 617 & 463 & $3571^{b}$ \\
\hline
\end{tabular}

AMA, advanced maternal age; MS-AFP, maternal serum AFP screen.

${ }^{a}$ Infertility, maternal infection, blood group incompatibility, etc.

${ }^{b}$ The total (3571) is greater than the number of patients seen (3085) because some patients were referred for more than one reason.

categories and percentages comprised the remainder of our referrals: positive family history, $8 \%(N=292)$; teratogen exposure, $6 \%(N=203)$; diabetes, $5 \%(N=173)$; hemoglobin mutation, $4 \%(N=134)$; other, $3 \%(N=114)$ (e.g., infertility, maternal infection, blood group incompatibility); pregnancy losses, $2 \%(N=79)$; and abnormal ultrasound, $2 \%(N=78)$. The referral reasons were similar to those found in all high risk Prenatal Diagnostic Centers (PDC) in California. This was determined by comparison with the Fiscal Year Summary reports for all PDC patients provided by the Genetic Disease Branch, California Department of Health Services.

\section{Acceptance of amniocentesis}

\section{Overall}

Of the 3085 patients, 1876 (61\%) were offered amniocentesis either for AMA, an MS-AFP-positive screen result, or an abnormal ultrasound, when the gestational age was between 15 and 22 weeks. For the remaining 1209 patients (39\%), amniocentesis was not indicated, or the pregnancy was too advanced ( $\geq 24$ weeks gestational age). Of the 1876 patients offered amniocentesis, 52\% $(N=967)$ accepted and $48 \%(N=909)$ declined. There was a statistically significant decline in the amniocentesis acceptance rate over the study period of 1995 2001: 63\% $(N=207)$ in 1995 to $39 \%(N=102)$ in $2001(P<$ $0.0001)$. There was a significantly higher acceptance rate by the African American patients $(63 \%, N=255)$ than the Hispanic patients $(48 \%, N=687)(P<0.0001)$ over the seven-year study period. A higher acceptance rate for African Americans was found consistently for each of the seven years of the study (Fig. 2).

\section{AMA population}

Of the total AMA population of 1491, 1337 (90\%) were offered amniocentesis. The excluded 154 patients were not offered the test because of a miscarriage or advanced gestational

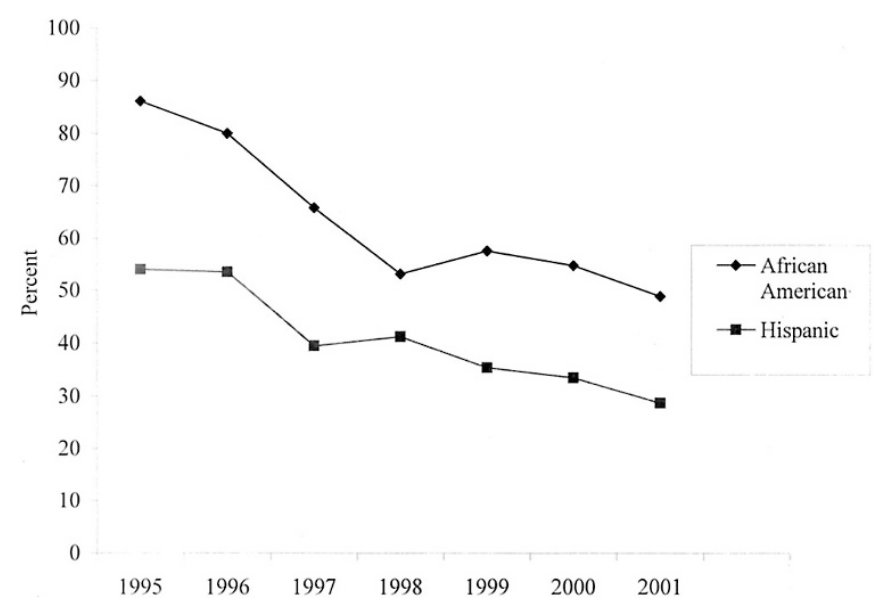

Fig. 2. Overall amniocentesis acceptance by ethnicity.

age ( $\geq 24$ weeks). Of the1337 offered the test, $46 \%(N=610)$ accepted and $54 \%(N=727)$ declined. The downward trend from a high of $60 \%(N=146)$ acceptance in 1995 to $34 \%(N=$ $54)$ in 2001 is significant $(P<0.0001)$. There was also a significant difference between acceptance by African Americans, $62 \%(N=157)$, and Hispanics, $41 \%(N=432)$. The higher acceptance rate for African Americans was consistent over the study period $(P<0.05)$. The average age of both groups was 38 years.

\section{MS-AFP-positive screen population}

A total of 1007 patients were referred because of a positive MS-AFP screen result for trisomy 21 , trisomy 18 , or neural tube defect (NTD). Of these, 726 (72\%) were referred because of positive Trisomy 21 or 18, and 281 (28\%) were positive for NTD, family history of NTD, or the use of valproic acid or carbamazepine, seizure medications known to be associated with NTD. (During the years of this study, women with a fam- 
ily history of an NTD or with teratogen exposure were automatically included in the California Expanded AFP Screening Program and consequently offered amniocentesis.)

After ultrasound dating, 514 (52\%) patients remained positive and were offered amniocentesis; $64 \%(N=331)$ accepted and $36 \%(N=183)$ declined. There was a statistically significant decline in acceptance over the years $(P<0.0001)$ (Fig. $3 \mathrm{~A})$. The difference between African American acceptance, $69 \%(N=88)$, and Hispanic acceptance, $63 \%(N=240)$, was not significant in the MS-AFP population.

\section{A}
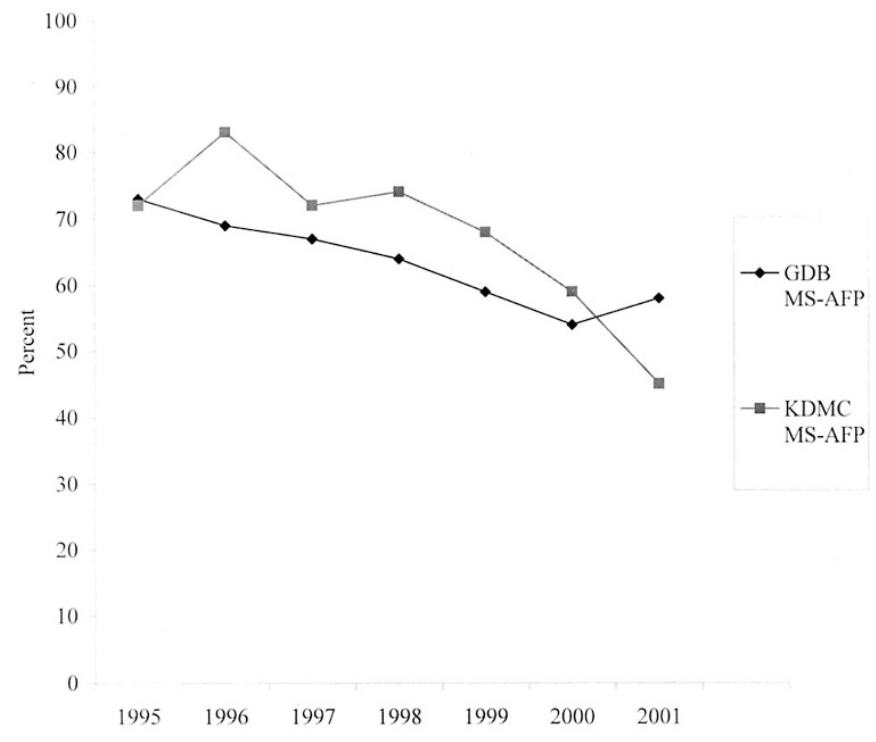

B

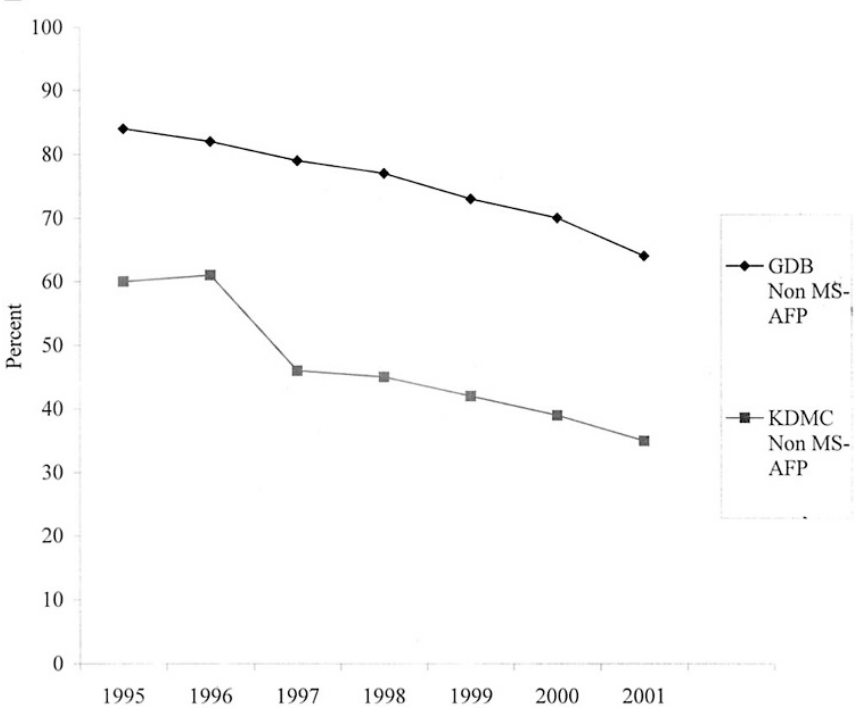

Fig. 3. A, Comparison of GDB and KDMC amniocentesis acceptance rates for MSAFP. B, Comparison of GDB and KDMC amniocentesis acceptance rates for non-MSAFP patients. KDMC, King Drew Medical Center; GDB, California Genetic Disease Branch; MS-AFP, maternal serum alpha-fetoprotein screen.

\section{Amniocentesis acceptance by age}

The acceptance rate of amniocentesis for all referral reasons among patients who were $<35$ years of age was $65 \%(N=$ 316). This is significantly higher than the amniocentesis acceptance rate of $47 \%(N=651)$ among those who were 35 years and older $(P<0.001)$. A comparison of the amniocentesis acceptance rate among non-MS-AFP patients by age showed a similar difference between those who were younger than 35 years, $59 \%(N=93)$, and those who were older, $45 \%(N=$ 537) $(P<0.001)$ (Table 2).

To avoid the possible age bias when looking at the total population and the non-MS-AFP population, for which the primary referral reason is AMA, we looked at the MS-AFPpositive population, which is not referred for age alone, to see if there was an age effect in this group. The rate of amniocentesis acceptance among the MS-AFP-positive patients who were $<35$ years was not found to be significantly higher, $68 \%$ $(N=223)$, than among those who were 35 years and older, $59 \%(N=114)$. However, when the two ethnic groups were looked at separately, the age-related difference in amniocentesis acceptance was significantly greater among the younger MS-AFP-positive Hispanic patients, $67 \%(N=159)$ versus $55 \%(N=81)(P<0.025)$, but not among African Americans, $68 \%(N=62)$ versus $72 \%(N=26)$ (Table 2$)$.

The acceptance rate among women 35 years and older was significantly higher in the MS-AFP positive group, 59\% ( $N=$ $114)$, than for the non-MS-AFP group, $45 \%(N=537)(P<$ $0.001)$, but the difference in acceptance for the two referral reasons was not significant in the younger age group, 68\% $(N$ $=223)$ versus $59 \%(N=93)($ Table 2$)$.

\section{Comparison with statewide amniocentesis acceptance}

The California Genetic Disease Branch (GDB) collects statewide data for both MS-AFP patients and non MS-AFP patients, but a break down by ethnicity is possible only for the MS-AFP. Non MS-AFP patient data includes the number of amniocentesis and CVS procedures offered and accepted by statewide PDC prenatal patients who are offered these procedures for advanced maternal age, family history of a genetic disorder, and other indications such as teratogen exposure or an abnormal ultrasound.

To compare our patients with the statewide data, we divided our patients into MS-AFP and non MS-AFP. Although not directly comparable with the Genetic Disease Branch data due to the differences in total numbers of patients in the two data sets, downward trends in overall acceptance are apparent in both our data and the statewide data (Fig. 3A and 3B).

The seven-year average of amniocentesis acceptance for our MS-AFP patients of $64 \%(N=331)$ is comparable to the statewide MS-AFP acceptance rate of $62 \%(N=65775)$. Our Hispanic amniocentesis acceptance rate was $63 \%(N=240)$, which is higher than the statewide Hispanic acceptance rate of $56 \%(N=26159)$. Our African American acceptance rate was $69 \%(N=88)$, which is higher than the statewide African American acceptance rate of $63 \%(N=5014)$. 
Table 2

KDMC amniocentesis acceptance by age group

\begin{tabular}{|c|c|c|c|c|c|}
\hline & Non-MS-AFP and MS-AFP ${ }^{a}$ & Non-MS-AFP ${ }^{a}$ & MS-AFP ${ }^{a}$ & Hispanic & African American \\
\hline No. amniocenteses offered to patients $<35$ years & 486 & 157 & 329 & 236 & 91 \\
\hline No. patients accepting $<35$ years & $316(65 \%)$ & $93(59 \%)$ & $223(68 \%)$ & $159(67 \%)$ & $62(68 \%)$ \\
\hline No. amniocenteses offered to patients $\geq 35$ years & 1390 & 1198 & 192 & 148 & 36 \\
\hline \multirow[t]{2}{*}{ No. patients accepting $\geq 35$ years } & $651(47 \%)$ & $537(45 \%)$ & $114(59 \%)$ & $81(55 \%)$ & $26(72 \%)$ \\
\hline & $P<0.001$ & $P<0.001$ & NS & $P<0.025$ & NS \\
\hline
\end{tabular}

${ }^{a}$ All including other and unknown ethnicities.

However, our non-MS-AFP overall acceptance rate of $47 \%$ $(N=636)$ is drastically lower than the statewide non-MS-AFP acceptance rate of $75 \%(N=227009)$. Our acceptance rate for non-MS-AFP Hispanic patients was $42 \%(N=447)$ and for African American patients the rate was $60 \%(N=167)$. The statewide data includes Caucasians and other ethnic groups not significantly represented in the KDMC population. It should be pointed out that Hispanics represented only $30 \%$ of the statewide non-MS-AFP population over the study period.

\section{Abnormalities}

There were $210(7 \%)$ abnormalities detected among the 3085 total patients referred for genetic counseling. Table 3 summarizes the abnormalities detected, either via ultrasound or amniocentesis. The abnormalities found were chromosome abnormalities $(N=48)$, ultrasound abnormalities $(N=158)$, and single gene defects $(N=4)$.

Ultrasound detected 12 fetuses with ventral wall defects, 21 with anencephaly, and 5 with spina bifida. Isolated anomalies such as echogenic foci, cleft lip, ascites, dilated kidneys, ventriculomegaly, cardiac defects, and limb defects were found in 82 fetuses. A fetal demise or missed abortion was detected in 38 patients.

Of the 967 patients who had an amniocentesis for AMA, MS-AFP, abnormal ultrasound, or other indications, 48 (5\%) fetuses were found to have chromosome defects: $3 \%$ were abnormal males $(N=28)$, and $2 \%$ were abnormal females $(N=$ 20). There were 29 autosomal trisomies: four trisomy 13, five trisomy 18, eighteen trisomy 21 (including two with Robertsonian translocations), and two trisomy 22 (including one mosaic trisomy 22). Of the five sex chromosome aneuploidies, two were mosaic. There were fourteen structural rearrangements; 13 were balanced translocations and one was unbalanced.

\section{DISCUSSION}

Our study of amniocentesis acceptance rates and abnormalities is unique because of our Hispanic and African American population ( $76 \%$ and $22 \%$ ), the relatively large number of patients (3085), and the number of years (1995-2001) included in the study. As mentioned previously, several studies have found a consistently lower amniocentesis acceptance rate among minority populations when compared to Caucasians in
Table 3

Abnormalities found by ultrasound and/or amniocentesis

\begin{tabular}{|c|c|}
\hline Abnormalities & Number \\
\hline \multicolumn{2}{|l|}{ Chromosome abnormalities } \\
\hline \multicolumn{2}{|l|}{ Autosomal trisomies } \\
\hline \multicolumn{2}{|l|}{ Chromosome } \\
\hline 13 & 4 \\
\hline 18 & 5 \\
\hline 21 & $\begin{array}{l}18 \text { (including } 2 \text { robertsonian } \\
\text { translocations) }\end{array}$ \\
\hline 22 & 2 (including 1 mosaic) \\
\hline \multicolumn{2}{|l|}{ Sex chromosomes } \\
\hline Aneuploidy & 3 \\
\hline Mosaics & 2 \\
\hline \multicolumn{2}{|l|}{ Structural rearrangements } \\
\hline Unbalanced & 1 \\
\hline Balanced & 13 \\
\hline \multicolumn{2}{|l|}{ Ultrasound Abnormalities } \\
\hline Ventral wall defects & 12 \\
\hline Fetal demise or missed abortion & 38 \\
\hline Other $^{a}$ & 82 \\
\hline \multicolumn{2}{|l|}{ Neural tube defects } \\
\hline Anencephaly & 21 \\
\hline Spina bifida & 5 \\
\hline Single Gene Defects & 4 \\
\hline Total & $210(7 \%)$ \\
\hline
\end{tabular}

${ }^{a}$ Echogenic foci, cleft lip, ascites, dilated kidney, ventriculomegaly, cardiac defects, and limb defects

the same study. ${ }^{1,5,8,9}$ California statewide MS-AFP data from 1995 to 2001 also reveals a significant difference between Caucasians and the Hispanic and African American ethnic groups: Caucasian MS-AFP acceptance was $67 \%(N=22925)$, whereas Hispanic acceptance was 56\% $(N=26159)$ and African American acceptance was $63 \%(N=5014)(P<0.0001)$. We did not have a sufficient number of Caucasian patients in our PDC to make a similar comparison. 
Statewide data for the non-MS-ASP population is not broken down by ethnicity; it shows an amniocentesis and CVS acceptance rate of $75 \%(N=227009)$ for all ethnicities 1995 2001. The overall acceptance rate among the KDMC non-MSAFP patients was $47 \%(N=636)$. We suspect that it is our proportionally larger Hispanic population that accounts for the much lower amniocentesis acceptance rate among our patients. Hispanics comprise $76 \%$ of our population, whereas they comprise approximately $30 \%$ of the PDC population statewide over the same period of time. Interestingly, however, the acceptance rate of amniocentesis by our Hispanic MS-AFP patients $(63 \%)$ is higher than the statewide acceptance rate for MS-AFP Hispanics (56\%).

\section{Amniocentesis acceptance by referral reasons, ethnicity, and age}

\section{Referral reasons}

The AMA population is our largest single referral group (Table 1). The overall amniocentesis acceptance rate of $46 \%$ for AMA referrals over the seven-year period is significantly lower than the $64 \%$ acceptance rate for our MS-AFP patients $(P<$ 0.0001). A possible explanation for this difference is that, unlike AMA patients who are assigned a risk based solely on age, patients with a positive MS-AFP screen have their screening results as evidence that their pregnancy is at risk for a serious problem.

The statewide data shows a significantly higher acceptance rate among non MS-AFP patients (75\%) than among the MSAFP PDC patients $(62 \%)(P<0.0001)$. As stated above, the reverse was true of our population.

\section{Ethnicity}

Overall there is a significantly higher rate of amniocentesis acceptance among our African American patients (63\%) compared to our Hispanic patients $(48 \%)(P<0.0001)$ (Fig. 2). However, although the difference between the two groups was significant for the AMA population $(62 \%$ vs. $41 \%)(P<$ $0.0001)$, it was not significant for the MS-AFP population (69\% vs. 63\%). The difference in acceptance of amniocentesis by the two ethnic groups cannot be attributed to an age difference, because the average age of both ethnic populations was not significantly different within the AMA and MS-AFP populations.

\section{Age}

The influence of age on the acceptance of amniocentesis is seen in the greater acceptance rate by patients who were younger than 35 years. Among the MS-AFP patients, this difference was not seen, except for the younger Hispanic patients. Our results indicate that the younger women in our PDC were more likely to accept amniocentesis independent of the referral reason, whereas the older women were more likely to accept amniocentesis because of an MS-AFP-positive screen (Table 2).

\section{Trends in amniocentesis acceptance rates}

A significant downward trend of amniocentesis acceptance over the seven-year period of 1995-2001 is seen for AMA, MSAFP, and non-MS-AFP populations in our PDC. The California statewide amniocentesis acceptance rates show similar declines in both the MS-AFP and non-MS-AFP populations (Fig. $3 \mathrm{~A}$ and $3 \mathrm{~B})$.

While the statewide non MS-AFP data are not reported by ethnicity, the statewide data does reveal that the proportion of Hispanics to non-Hispanics has increased from $27 \%$ in 1995 1996 to $32 \%$ in 2001-2002. The overall recent decline in acceptance of amniocentesis has been attributed by some to the increasing number of Hispanic births in California. However, the decline in amniocentesis acceptance is not limited to Hispanics, because statewide data show a downward trend in all ethnic groups. At our PDC, both ethnic groups show a downward trend.

\section{Abnormalities}

All patients referred to the PDC are considered to have highrisk pregnancies. Therefore, we would expect to see a larger number of prenatal abnormalities than would be seen in a general prenatal care clinic. Older mothers are known to have an increased risk of chromosome abnormalities and this is our largest referral group. Also, because Hispanics have been found to have a higher incidence of neural tube defects (NTD), we would expect to see a larger number of cases of anencephaly and spina bifida in our PDC.

The California Birth Defects Monitoring Program ${ }^{10}$ reports that about $3 \%$ of children $(1 / 33)$ are born with a birth defect, including structural defects and chromosome abnormalities. The $7 \%$ incidence in our clinic is more than twice this general population risk. Of 3085 high-risk patients seen in our PDC over the seven-year period, we had 26 NTD cases (all among Hispanic patients), 21 cases of anencephaly, and 5 of spina bifida. This NTD frequency of 1 in 119 is 17 times greater than the frequency for Hispanics reported by the California Birth Defects Registry among live births and fetal deaths in California for the years 1997-2001. The frequency of anencephaly (1/147) among our patients was 34 times, and spina bifida (1/617) was 4 times the respective frequencies for Hispanics statewide. The frequency of ventral wall defects in our clinic was 1 in 257, which is 10 times the frequency reported among Hispanics and African Americans in California live births. The frequency of chromosome abnormalities identified among our clinic patients was 1 in 64, more than 3 times the California statewide frequency of 4.7 in 1000 found among live births and fetal deaths for women 34 years and older.

\section{Commentary}

The majority of our patients have health coverage through the California MediCal program; therefore, any lower rates of acceptance at our PDC cannot be attributed to financial factors, as was found in one study. ${ }^{11}$ 
Language was probably not a major factor in the decision about amniocentesis at our PDC because both groups were counseled in their native language. However, the fact that the counselor was not of the same ethnicity of either group could have been a factor. African Americans were counseled without the presence of a third person, and one might argue that the more intimate counseling session may have contributed to their higher rate of acceptance.

Although it is possible that the counseling by the $\mathrm{PhD}$ Medical Geneticist at our PDC is different from that provided by a trained MS Genetic Counselor at other PDCs, we do not think it is significantly different. Our counselor worked with and participated in counseling sessions with several different MS Genetic Counselors during her training. The counseling sessions at our PDC are similar in structure. Although one could argue that this might be a factor in the higher rate of acceptance for MS-AFP in our population compared to statewide data, it would not explain the downward trend in acceptance, because this downward trend was paralleled by the state data of patients counseled primarily by MS Genetic Counselors.

The focus of this study was solely to determine the characteristics of our PDC population and their acceptance of amniocentesis. We did not attempt any systematic study of the reasons why our patients accept or decline amniocentesis. However, patients often comment on their decisions for accepting or declining during the counseling session. Comments are often about their opposition to terminating a pregnancy for any reason, their unwillingness to risk a miscarriage, and their fear that the needle will harm the baby. When offered the amniocentesis, Hispanic patients often say, "Esta en las manos de Dios" ("no, because it is in God's hands").

In the counseling session, Hispanic patients often refer to amniocentesis as "the needle" test. Even after seeing a video in Spanish that describes the procedure, as well as the various reasons one might be offered an amniocentesis, a significant number of patients have the incorrect idea that the needle is inserted into the navel and that the needle will touch the baby. It is important to note that for many of our patients, the counseling session is the first time they have heard of the procedure of amniocentesis, although almost all patients have previous knowledge of prenatal ultrasound examinations.

The MS-AFP-positive patients often do not know they have had the screen until they come for the genetic counseling and ultrasound followup. It is common for these patients to check "no" on the questionnaire that asks if they have had the Expanded MS-AFP screen. When asked by the counselor if they remember signing the booklet, a considerable number do not. Even if they do remember, they are usually unaware that the screen is not a test and that only an amniocentesis will tell them for certain whether the fetus is abnormal. AMA patients who decline amniocentesis and then become MS-AFP screen positive are often surprised to find they are being offered an amniocentesis a second time. It is important that prenatal clinic staff have an accurate understanding of the maternal serum screening program and the possible reasons for a positive screen result. The referring clinic staff needs to explain that the MS-AFP is a screen and not a test and that an amniocentesis may be offered. Lack of understanding by the clinical contact can be a source of misinformation and needless anxiety for the pregnant patient.

The reasons behind the ethnic differences in amniocentesis acceptance and the downward trend over the sevenyear period would be interesting to explore further. Because of the increasing numbers of Hispanics in California and across the nation, there is a need for more health care providers who speak Spanish and know the culture. There is also a need for public education on the MS-AFP screening program, which appears, in general, to be more acceptable among prenatal patients because it is noninvasive. Another need is for ultrasound studies by well-trained personnel in the smaller clinics. This would increase the accuracy of gestational dating, critical to the accuracy of the MS-AFP screening results, and also assist in the early detection of prenatal abnormalities. Because the current trend is for patients to go to private doctors who accept MediCal rather than to county/university hospital obstetric clinics, it is necessary for doctors to refer pregnant patients early in the pregnancy to facilities where there are high-quality ultrasound equipment and experienced ultrasound technicians. These are greater problems in underserved minority communities.

The high rate of chromosome abnormalities, structural defects, and neural tube defects in the KDMC PDC warrants attention and further study. However, it may be a reflection of the high-risk clientele served in our hospital. The larger number of neural tube defects in our predominantly foreign-born Hispanic population might be expected, because it is known that these defects occur in higher frequency in this group. It is encouraging to note that many of the patients were aware from public service announcements of the protective effects of folate. Some patients report taking folate before becoming pregnant. Therefore, public health announcements, especially on television and in Spanish (and other languages for other populations), can have a positive impact on the improvement of prenatal care.

\section{ACKNOWLEDGMENTS}

This research was supported in part by NIH MBRS R25GM62252, RCMI Grant no. G12RR03026-17, and the Genetic Disease Testing Fund of the State of California, Department of Health Services, Genetic Disease Branch.

\section{References}

1. Tercyak KP, Johnson SB, Roberts SF, Cruz AC. Psychological response to prenatal genetic counseling and amniocentesis. Patient Educ Couns 2001;43:73-84.

2. Gilbert L, Nicholl J, Alex S, Smetherst I, Mander A, Andrews A. Ethnic differences in the outcome of serum screening for Down's syndrome. BMJ 1996;312:94-95.

3. Ford C, Moore AJ, Jordan PA, Bartlett WA, Wyldes MP, Jones AF et al. The value of screening for Down's syndrome in a socio-economically deprived area with a high ethnic population. BrJ OB Gyn 1998;105:855-859. 


\section{Baker et al.}

4. Marion JP, Kassam G, Fernhoff PM, Brantley KE, Carroll J, Klein L et al. Acceptance of amniocentesis by low-income patients in an urban hospital. Am J Obstet Gynecol 1980;138:11-15.

5. Kocun CC, Harrigan JT, Canterino JC, Feld SM, Fernandes CO. Changing trends in patient decisions concerning genetic amniocentesis. Am J Obstet Gynecol 2000;5: 1018-1020.

6. Kupperman M, Gates E, Washington AE. Racial-ethnic differences in prenatal diagnostic test use and outcomes: Preferences, socioeconomics, or patient knowledge? Obstet Gynecol 1996;87:675-682.

7. Lowry DLB, Campbell SA, Krivchenia EL, Dvorin E, Duquette K, Evans MI. Impact of abnormal second-trimester maternal serum single, double, and triple screening on patient choices about prenatal diagnosis. Fetal Diagn Ther 1995;10:286-289.
8. Marini T, Sullivan J, Naeem R. Decisions about amniocentesis by advanced maternal age patients following maternal serum screening may not always correlate clinically with screening results: Need for improvement in informed consent process. Am J Med Genet 2002;109:171-175.

9. Marteau TM, Kidd JH, Cook R, Michie S, Johnston M, Slack J et al. Perceived risk not actual risk predicts uptake of amniocentesis. Br J OB Gyn 1991;98:282-286.

10. Birth Defects Registry: The California Birth Defects Monitoring Program, California Department of Health Service and the March of Dimes Birth Defects Foundation, 1997-2001. Available at: http://www.cbdmp.org.

11. Julian-Reynier C, Macquart-Moulin G, Moatti JP, Aurran Y, Chabal F, Ayme S Reasons for women's non-uptake of amniocentesis. Prenat Diagn 1994;14:859864. 